\title{
Marian Hobson, Diderot and Rousseau: networks of Enlightenment
}

Eszter Kovács

\section{(2) OpenEdition}

1 Journals

Édition électronique

URL : https://journals.openedition.org/studifrancesi/4696

DOI : 10.4000/studifrancesi.4696

ISSN : 2427-5856

Éditeur

Rosenberg \& Sellier

Édition imprimée

Date de publication : 1 avril 2012

Pagination : 150-151

ISSN : 0039-2944

\section{Référence électronique}

Eszter Kovács, « Marian Hobson, Diderot and Rousseau: networks of Enlightenment », Studi Francesi [En ligne], 166 (I | LVI) | 2012, mis en ligne le 30 novembre 2015, consulté le 19 novembre 2021. URL :

http://journals.openedition.org/studifrancesi/4696 ; DOI : https://doi.org/10.4000/studifrancesi.4696

Ce document a été généré automatiquement le 19 novembre 2021.

\section{(c) $(1) \&$}

Studi Francesi è distribuita con Licenza Creative Commons Attribuzione - Non commerciale - Non opere derivate 4.0 Internazionale. 


\title{
Marian Hobson, Diderot and Rousseau: networks of Enlightenment
}

\author{
Eszter Kovács
}

\section{RÉFÉRENCE}

MARIAN HOBSON, Diderot and Rousseau: networks of Enlightenment, edited by Kate E. TUNSTALL and Caroline WARMAN, Oxford, Voltaire Foundation («SVEC» 2011/04), 2011, pp. 366.

1 Ce volume réunit quatorze articles de Marian Hobson, écrits entre 1973 et 2005, traduits en anglais par les éditrices. Les articles sont classés dans cinq unités thématiques après l'article le plus récent qui sert d'introduction: deux articles sont consacrés au Paradoxe sur le comédien, trois au Neveu de Rameau, deux à la question de la causalité, trois à l'esthétique et trois au problème de la proportion.

Dans l'article From Diderot to Rousseau via Rameau (pp. 15-29), Marian Hobson observe les différences entre les deux auteurs dans leur postérité, dans leur rapport à la religion, à la temporalité et au langage: elle avance que le dialogue entre Lui et Moi dans Le Neveu évoque les discussions entre Diderot et Rousseau avec un regard rétrospectif sur leur amitié. Dans The "Paradoxe sur le comédien" is a paradox (pp. 33-64), Hobson reformule l'idée débattue dans le texte: la tragédie française n'est pas naturelle et doit être rejetée; l'art n'est pas naturel mais ne doit pas être rejeté. Elle analyse le Paradoxe comme un réseau d'antithèses à tout niveau, qui reste toujours un paradoxe puisqu'il ne peut pas être résolu. Dans Sensibility and spectacle: the medical context for the "Paradoxe" (pp. 65-90), elle prouve que la définition que Diderot donne de la sensibilité s'intègre dans les débats scientifiques de l'époque. Diderot se réfère à la discussion autour de la sensibilité et l'irritabilité en considérant le contrôle de soi de l'acteur. Dans Pantomime, spasm and parataxis: "Le Neveu de Rameau" (pp. 93-113), elle examine le procès spasmodique chez Lui pour conclure que le volontaire et l'involontaire fonctionnent d'une manière atypique chez ce "parasite» et la parataxe devient la projection linguistique de ce phénomène. Dans Deictics and dialectics in "Le Neveu de Rameau" 
(pp. 115-125), elle analyse la situation d'énonciation dans le dialogue: les renversements des points de vue prouvent la subjectivité du sujet parlant. Lists, parataxis and "Le Neveu de Rameau" (pp.127-139) continue l'analyse linguistique de l'article précédent: cette approche contribue à l'interprétation du personnage de Rameau, caractérisé par l'expression paratactique. Dans "Jacques le fataliste": the art of the probable (pp. 143-163), Hobson observe les concepts du hasard et de la nécessité, du possible et du probable à travers l'inconséquence de l'individu et de la narration dans le roman. L'article "Nexus effectivus" and "nexus finalis": causality in Rousseau's "Discours sur l'inégalité" and the "Essai sur l'origine des langues" (pp.165-199) examine les différences entre les causes actuelles en jeu dans le deuxième Discours et la cause singulière et divine qui se dégage de l'Essai. Dans Philosophy and Rococo style (pp. 203-212), Hobson lie la notion de l'instabilité et le style rocaille: l'irrégularité du style représente l'instabilité de la nature. Dans Diderot's "Lettre sur les sourds et muets": language and labyrinth (pp. 213-259), elle regarde minutieusement comment la Lettre s'intègre dans et se distingue de la philosophie du langage de l'époque. Les deux questions majeurs sont l'inversion-existe-il un ordre naturel de la pensée, un ordre préétabli à la langue ou l'ordre s'impose?-et la traduction - quelle est la base sémantique de la langue? Dans Kant, Rousseau and music (pp. 261-280), elle affirme que, bien qu'on ne puisse pas parler de filiation, certaines idées de Rousseau se retrouvent chez Kant mais dans une démonstration différente: pour Kant, la musique, insaisissable, est une expression et non pas une imitation. Dans Architecture, analogy and proportion (pp. 283-294), Hobson démontre que Diderot applique les débats autour de la proportion à la perception humaine et aux arts en général. Dans How to take the measure of a character (pp. 295-315), elle constate l'influence de Hogarth sur Diderot, selon lequel la proportion n'est pas absolue mais due à une perception dynamique. Dans Measuring statues, or special neutrality (pp. 317-331), elle observe les proportions idéales du XviII ${ }^{\mathrm{e}}$ siècle: pour le physionomiste Pieter Camper la beauté est un concept social et non pas une donnée naturelle.

Le livre de Marian Hobson n'est ni une lecture rapide ni une lecture facile. Les études mobilisent de nombreux domaines, de la linguistique à la médecine, de la philosophie du langage à la psychologie, de l'esthétique à l'épistémologie. L'auteure cherche à comprendre le texte dans son contexte large, entouré des polémiques qui le font naître: elle franchit ainsi les frontières des disciplines. Les associations complexes ou même inattendues peuvent parfois nous surprendre: on comprend leur rôle à la fin de l'article ou, encore mieux, lors d'une relecture. Nous aimerions souligner la grande qualité de l'ouvrage, qui nous permet de dépasser les limites de nos propres recherches et de comprendre un autre réseau que celui auquel nous étions habitués. 